\title{
Aufkommen und Zusammensetzung von Makrokunststoffen an der Donau
}

\author{
Johannes Mayerhofer · Sabine Lenz · Gudrun Obersteiner
}

Online publiziert: 6 . August 2020

(c) Der/die Autor(en) 2020

\section{Zusammenfassung Weltweit ist die Nachfrage nach Kunststoffen ungebro- chen. Der Werkstoff ist in beinahe allen \\ Occurrence and composition of macro-plastic waste along the Danube} Lebensbereichen anzutreffen. Mit den zunehmenden Produktionszahlen geht auch ein verstärkter Eintrag von Kunststoffen in die Umwelt einher. Besonders in Bezug auf die Verschmutzung der Weltmeere ist Plastikmüll heutzutage in aller Munde. Flüsse gelten dabei als die Haupteintragspfade der marinen Verschmutzung (z.B. Great Pacific Garbage Patch). Dennoch sind Quellen und Herkunft der Plastikabfälle sowie Transportwege und Umweltauswirkungen in fluvialen Systemen großteils unbekannt. Das liegt nicht zuletzt daran, dass vereinheitlichte Monitoringmethoden bisher fehlen. Das Projekt „PlasticFreeDanube“ setzt genau hier an. Ziel ist der Aufbau einer fundierten Wissensbasis zu Aufkommen und $\mathrm{Zu}$ sammensetzung von Kunststoffabfällen in und entlang der Donau. Daneben steht die Analyse von Transportverhalten und Umwelteinflüssen im Fokus. Der folgende Artikel befasst sich mit der Methodenentwicklung zur standardisierten Probenahme und Sortierung von Kunststoffabfällen in Flusssystemen sowie den Ergebnissen bisheriger Sortieranalysen im Projektgebiet des Nationalparks Donau-Auen.

\section{Schlüsselwörter}

Kunststoffverschmutzung · Eintrag über Flüsse · Nationalpark DonauAuen National Park.
Abstract Worldwide the demand for plastics is still unbroken. The material can be found in almost all areas of our daily life. The increasing production numbers are accompanied by an increased input of plastics into the environment. Plastic waste is on everyone's lips these days, especially with regard to the pollution of the oceans. Rivers are regarded as the main input paths for marine pollution (e.g. Great Pacific Garbage Patch). However, sources and origins of plastic waste as well as transport routes and environmental impacts in fluvial systems are largely unknown. This is not least due to the lack of standardized monitoring methods. The aim of the EDRF funded project PlasticFreeDanube is therefore to establish a sound knowledge base on the occurrence and composition of plastic waste in and along the Danube River. In addition, the analysis of transport behaviour and environmental influences is also in focus. The following article deals with the development of methods for standardised sampling and sorting of plastic waste in river systems as well as the results of sorting analyses in the project area of the Donau-Auen

Keywords Plastic pollution - Riverine input - Danube - Donau-Auen Nationalpark

\section{Einleitung}

Kunststoff (KS) gilt als der Werkstoff des 20. und 21. Jahrhunderts. Durch seine universellen technischen Eigenschaften hat er sich in fast allen Bereichen unseres Lebens als wichtiges Material durchgesetzt. Die weltweite Kunststoffproduktion liegt derzeit bei knapp 350 Mio. Tonnen und ist in den letzten 50 Jahren um mehr als $200 \%$ angestiegen (PlasticsEurope 2018). Gleichzeitig haben sich Kunststoffabfälle zu einem globalen Umweltproblem entwickelt, das besonders marine Ökosysteme belastet.

Weltweite Schätzungen zu Kunststoffabfällen, die über Flüsse in die Ozeane gelangen, schwanken teils stark in der Literatur. Die angegebenen jährlichen Eintragsmengen reichen von 0,4 bis 12,7 Mio. $\mathrm{t}$ bzw. 2 bis $10 \%$ der weltweiten Jahresproduktionsmenge (Vannela 2012; Jambeck et al. 2015; Lebreton et al. 2017; Schmidt et al. 2017). Dennoch wird weithin davon ausgegangen, dass etwa $80 \%$ der marinen Vermüllung vom Land her stammen. Flüsse zählen dabei als die Hauptverursacher der Verschmutzung (GESAMP 1990; Jambeck et al. 2015). Quellen und Wege sowie die Umweltauswirkungen in Flüssen und der fluvialen Umwelt sind jedoch weitgehend ungeklärt. Die meisten der aktuell vorhandenen Studien fokussieren auf Mikrokunststoffe ( $\leq 5 \mathrm{~mm}$ ). Außerdem verwenden frühere Studien zum Aufkommen und Herkunft von Kunststoffabfällen in Flüssen unterschiedliche Methoden zur Probenahme, Messung und Sortierung und sind daher weitgehend nicht vergleichbar. Zuverlässige Schätzungen sind jedoch unerlässlich, um Maßnahmen zur Verringerung der Kunststoffverschmutzung zu entwickeln und Erfolge messund sichtbar zu machen. Aus diesem Grund zielt das grenzüberschreitende Projekt „PlasticFreeDanube“, das vom Europäischen Fonds für regionale Entwicklung im Rahmen von Interreg Slowakei-Österreich kofinanziert wird, darauf ab, neue Erkenntnisse zu diesem Thema für die Donau zu gewinnen. Geprägt durch das größte grenzüberschreitende europäische Flussgebiet stellt die Donau ein optimales Projektgebiet dar. Als Hauptschlagader hat die Donau einen direkten und bedeutenden Einfluss auf das Schwarze Meer. Lechner et al. (2014) schätzen die jährliche Menge an Kunststoffen, die über die Donau ins Schwarze Meer gelangen, auf 1530 t (mit einem stärkeren Fokus auf 
Mikrokunststoff). Informationen über die Menge und Zusammensetzung von Makrokunststoff ( $>5 \mathrm{~mm}$ ) fehlen noch.

\section{Projektgebiet und Ziele}

Das Untersuchungsgebiet umfasst die Donau, einschließlich des angrenzenden Donauraums ( $\mathrm{HQ}_{30}$-Überschwemmungsflächen) vom Ballungsraum Wien über Bratislava bis hinunter zum slowakischen Wasserkraftwerk (WKW) Gabčíkovo. Damit erstreckt sich das Projektgebiet über fast 100 Flusskilometer (WKW Freudenau 1921 bis WKW Gabčíkovo 1823).

Mit mehr als 9600 ha und einer Länge von ca. $36 \mathrm{~km}$ stellt der Nationalpark Donau-Auen die letzte noch verbliebene große Feuchtlandschaft Mitteleuropas dar. Die frei fließende Donau unterscheidet sich zum Teil erheblich von den anderen Flussabschnitten in Österreich. Entscheidend ist die dynamische Veränderung des Wasserspiegels, der teilweise mehr als $7 \mathrm{~m}$ betragen kann, und die daraus resultierende ständige Erneuerung und Neugestaltung der Auenlandschaft (NPDA 2011).

Von bisherigen Freiwilligensammlungen im Nationalpark ist bekannt, dass das Aufkommen an Kunststoffen entlang des Flusses sehr unterschiedlich ist. In einigen Abschnitten des Nationalparks können punktuelle Akkumulations-Hotspots beobachtet werden. Innerhalb von wenigen Quadratmetern treten hier vermehrt Plastikablagerungen auf, während in den umliegenden bzw. angrenzenden Flächen kein Plastikmüll aufzufinden ist. Darüber hinaus werden üblicherweise Gebiete von den Kunststoffverschmutzungen befreit, die für die Sammelteams leicht zugänglich sind. Dadurch kommt es aber international zu einer Verfälschung der Ergebnisse, da aufgrund der unterschiedlichen räumlichen Gegebenheiten keine Aussagen über das tatsächliche Kunststoffaufkommen entlang eines Flusses getroffen werden können.

Ziel des Projektes PlasticFreeDanube ist es daher, durch standardisierte Sammel- und Sortierprotokolle einen Vergleich von Ergebnissen in Bezug auf Kunststoffaufkommen und -zusammensetzung an Flüssen zu ermöglichen. Daneben soll die aktuelle Zusammensetzung der Kunststoffe im Projektgebiet analysiert werden, um entsprechende Vermeidungsstrategien zu entwickeln.

\section{Methode}

Im Rahmen einer umfassenden Literaturrecherche wurden bestehende Leitfäden, Empfehlungen, Kataloge und Berichte zur Überwachung und zum Monitoring von (marinen) Kunststoffverschmutzungen ausgewertet. Mithilfe der gewonnenen Erkenntnisse wurde ein für die gegenständliche Fragestellung geeignetes Probenahme- und Sortierprotokoll entwickelt. Folgende Voraussetzungen wurden dabei berücksichtigt:

- Um ein langfristiges Monitoring zu gewährleisten, ist die Umsetzung der Sammlung und Sortierung durch Laien (im Rahmen von Freiwilligensammlungen) zu gewährleisten.

- Die Ergebnisse der Sortierungen sollen Auskünfte über die Herkunft der Kunststoffe ermöglichen, um entsprechende Vermeidungsmaßnahmen setzen zu können.

- Mithilfe des Sammelprotokolls wird die Abschätzung zur potenziellen
Wahrscheinlichkeit von Kunststoffabfällen ermöglicht, sodass in Abhängigkeit von z.B. Uferstruktur, Hinterland und Größe des Sammelgebietes Ergebnisse unterschiedlicher Standorte verglichen werden können.

Die entwickelten Sammel- und Sortierprotokolle wurden in weiterer Folge mithilfe von Freiwilligensammlungen auf ihre Praktikabilität getestet und aufgrund der gewonnenen Erkenntnisse adaptiert. Ergebnis dieses Implementierungsprozesses ist ein standardisiertes Probenahme- und Sortierprotokoll von Kunststoffabfällen in und entlang von Flusssystemen.

Daneben wurde die Zusammensetzung der Kunststoffabfälle im Gebiet des Nationalparks Donau-Auen detailliert untersucht.

\subsection{Probenahme}

Zur Bestimmung der Herkunft der Kunststoffabfälle und ihrer ungefähren Menge in und entlang der Donau wurden verschiedene Ansätze gewählt. Zum einen wurden gesammelte Kunststoffabfälle aus Sammelaktionen von Freiwilligen verwendet, um Informationen über die Zusammensetzung des Abfalls zu erhalten. Andererseits wurden standardisierte Probenahmeverfahren (sowohl in der Uferzone als auch im Hinterland der $\mathrm{Au}$ ) entwickelt, um Kunststoffe in regelmäßigen Abständen zu sammeln, zu verwiegen, zu zählen und einer bestimmten Größenklasse zuzuordnen, um Aussagen über das Transportverhalten von Kunststoffen im Flusssystem zu gewinnen. In diesem Artikel werden nur die Ergebnisse der Freiwilligen-Sammlungen diskutiert.

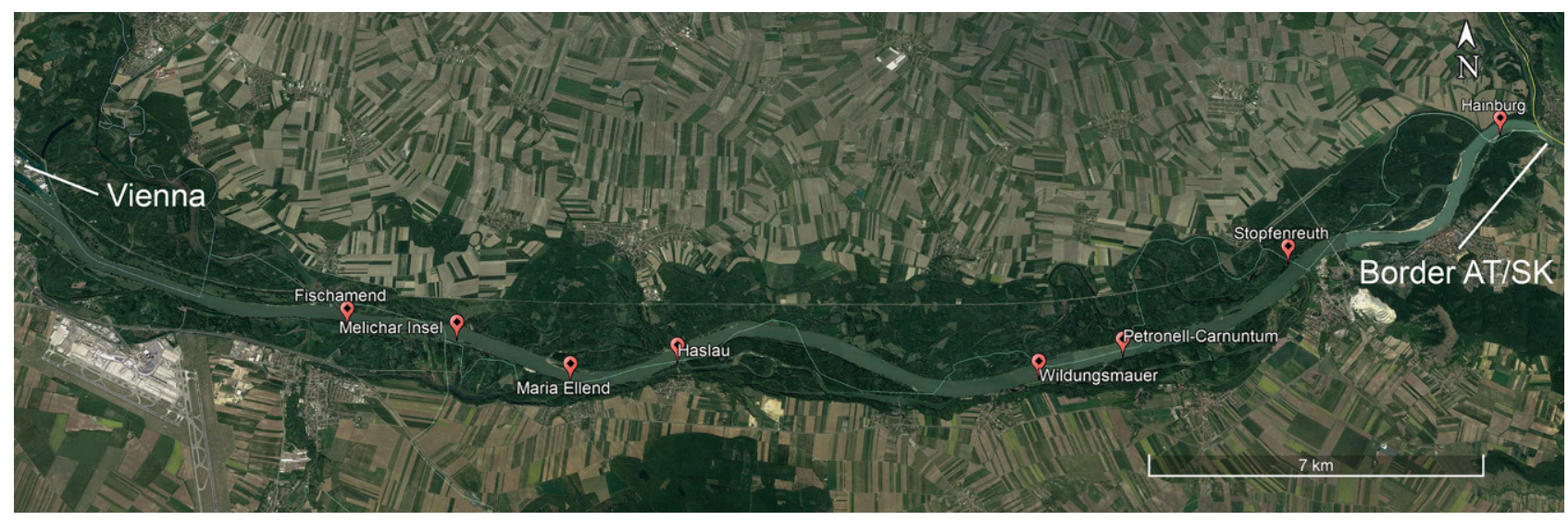

Abb. 1 Kartenausschnitt Nationalpark Donau-Auen mit Probenahmegebieten (rote Markierungen) 
Die Beprobung der Kunststoffabfälle wurde auf österreichischer Seite in Zusammenarbeit mit dem Projektpartner Nationalpark Donau-Auen (NPDA) durchgeführt. Gemeinsam mit Freiwilligen (Schulklassen, Firmen, Vereine etc.) wurden alle Abfälle je nach Gruppengröße, Durchschnittsalter, Witterungsbedingungen und Vegetation in unterschiedlichen Abschnitten bzw. Gebieten gesammelt. Dabei wurde meist bereits bei der Sammlung der Plastikmüll von anderen (Rest-)Abfällen separiert. Die acht Hauptsammelstellen (Abb. 1) wurden aufgrund jahrelanger Beobachtungen und Erfahrungen der NPDA-Ranger ausgewählt, da hier regelmäßig größere Kunststoffablagerungen auftreten.

Die Sammelkampagnen stellen eine Vollerhebung in den beprobten Gebieten dar. In Abhängigkeit von der Gruppengröße und der zur Verfügung ste- henden Zeit reinigen die Sammelteams in der Regel eine „definierte“ Länge (bis zu mehrere hundert Meter) neben dem Fließgewässer. Das umfasst die direkte Uferzone (50-100 m).

\subsection{Sortieranalysen}

Im Hinblick auf das Monitoring von anthropogener Meeresverschmutzung gibt es bereits eine Vielzahl von Abfallklassifizierungen (Cheshire et al. 2009; OSPAR 2010; van der Wal et al. 2015). Diese dienten als Ausgangspunkt für eine Gegenüberstellung der bestehenden Kataloge und der darin definierten Kunststofffraktionen. Um die Eintragspfade von Kunststoffen zu bestimmen und Vermeidungsmaßnahmen zu entwickeln und zu bewerten, ist es notwendig, Kunststoffabfälle zu klassifizieren.
Bei den Sortieranalysen erfolgt die Zuordnung der Kunststoffteile zu den jeweiligen Fraktionen (Tab. 1) - dem generellen Ziel des Projekts folgend, eine leicht von Freiwilligen anwendbare Methode zu schaffen - rein visuell, sprich mit „freiem Auge“. Im Fall der geschäumten Kunststoffe (z. B. Styroporteile) wurde neben der optischen Zuteilung durch bestimmte Merkmale (Korngröße, Farbgebung etc.) zusätzlich auf die Analysemethode der Röntgenfluoreszenzanalyse (XRF) zurückgegriffen. Oftmals handelt es sich um bereits stark verwitterte und kleinere Schaumstoffteile, deren ursprünglicher Verwendungszweck nicht identifiziert werden kann. Durch die (stichprobenartige) Bestimmung der Brom-Konzentration können Flammhemmer detektiert und so eine Unterscheidung zwischen Ver-

Tab. 1 PlasticFreeDanube Master List-Kategorisierung und Unterteilung von Kunststoffabfällen

\begin{tabular}{|c|c|c|c|c|c|}
\hline Hauptgruppe & Untergruppe & $\mathrm{Nr}$. & Kategorie & $\mathrm{Nr}$. & Unterkategorie \\
\hline \multirow{12}{*}{$\begin{array}{l}\text { Verpackung } \\
\text { (VP) }\end{array}$} & Getränkeflaschen & 1 & PET Getränkeflaschen inkl. Verschlüsse & - & - \\
\hline & \multirow{4}{*}{$\begin{array}{l}\text { Lebensmittel- } \\
\text { verpackung }\end{array}$} & \multirow[t]{4}{*}{2} & \multirow[t]{4}{*}{ Lebensmittelverpackung } & $2 a$ & Flexible LM-Verpackung \\
\hline & & & & $2 b$ & Hartplastik-Behälter \\
\hline & & & & $2 c$ & Getränkeverbundkartons \\
\hline & & & & $2 d$ & Geschäumte LM VP \\
\hline & \multirow{7}{*}{$\begin{array}{l}\text { Sonstige } \\
\text { (Nicht-Lebensmittel) } \\
\text { Verpackungen }\end{array}$} & 3 & Einweggeschirr & - & - \\
\hline & & 4 & Einkaufstragetaschen & - & - \\
\hline & & 5 & Kosmetikbehälter & - & - \\
\hline & & 6 & Reinigungsbehälter & - & - \\
\hline & & 7 & VP von Baustellenartikel & - & - \\
\hline & & 8 & Verpackungsfolien & - & - \\
\hline & & 9 & Sonstige Kunststoffverpackung & - & - \\
\hline \multirow[t]{3}{*}{ Geschäumt } & \multirow{3}{*}{$\begin{array}{l}\text { Geschäumte } \\
\text { Kunststoffe }\end{array}$} & \multirow[t]{3}{*}{10} & \multirow{3}{*}{$\begin{array}{l}\text { Geschäumter Kunststoff (Verpackung/ } \\
\text { Isolierung/geschäumte Füllstoffe/nicht } \\
\text { definierbare Schaumstoffartikel) }\end{array}$} & $10 \mathrm{a}$ & Expandiertes Polystyrol (EPS) für Verpackung \\
\hline & & & & $10 \mathrm{~b}$ & $\begin{array}{l}\text { Expandiertes oder extrudiertes Polystyrol (EPS/XPS) } \\
\text { für Dämmung }\end{array}$ \\
\hline & & & & $10 \mathrm{c}$ & Sonstige geschäumte KS \\
\hline \multirow{16}{*}{$\begin{array}{l}\text { Nicht- } \\
\text { Verpackung } \\
\text { (NVP) }\end{array}$} & \multirow{6}{*}{$\begin{array}{l}\text { Haushalt/Spiel/Sport/ } \\
\text { Freizeit }\end{array}$} & 11 & Haushalts(ähnliche)waren & - & - \\
\hline & & 12 & Kleidung, Schuhe und Accessoires & - & - \\
\hline & & 13 & Spiel-, Sport- \& Freizeitartikel & - & - \\
\hline & & 14 & Fischereizubehör & - & - \\
\hline & & 15 & Zigarettenartikel & - & - \\
\hline & & 16 & Feuerzeuge & - & - \\
\hline & \multirow{5}{*}{$\begin{array}{l}\text { Sanitär- \& } \\
\text { Medizinische Abfälle }\end{array}$} & \multirow[t]{4}{*}{17} & \multirow[t]{4}{*}{ Sanitärabfälle } & $17 a$ & Damenhygieneartikel \\
\hline & & & & $17 \mathrm{~b}$ & Feucht-/Reinigungstücher \\
\hline & & & & $17 \mathrm{c}$ & Wattestäbchen \\
\hline & & & & $17 d$ & Sonstige Sanitärabfälle \\
\hline & & 18 & Medizinische \& pharmazeutische Abfälle & - & - \\
\hline & \multirow[t]{4}{*}{ NVP Kunststoffe } & 19 & Seile und Schnüre & - & - \\
\hline & & 20 & Klebebänder & - & - \\
\hline & & 21 & Schifffahrtsgegenstände & - & - \\
\hline & & 22 & Fahrzeugteile & - & - \\
\hline & Bauabfall & 23 & Baustellenabfälle & - & - \\
\hline Sonstige & $\begin{array}{l}\text { Sonstige Kunststoff- } \\
\text { teile }\end{array}$ & 24 & $\begin{array}{l}\text { Nicht identifizierbare oder zuordenbare } \\
\text { Kunststoffteile }\end{array}$ & - & - \\
\hline
\end{tabular}


Tab. 2 Bisher im Rahmen des Projektes durchgeführte Freiwilligen-Sammlungen im NP Donau-Auen

\begin{tabular}{|c|c|c|c|c|c|c|c|}
\hline Jahr & $\Sigma$ Events & Teilnehmer & $\Sigma$ Strom-Kilometer & Kum. Sack-Volumen [hl] & $\begin{array}{l}\sum \text { Plastik } \\
{[\mathrm{kg}]}\end{array}$ & $\Sigma$ Rest-Abfall [kg] & $\begin{array}{l}\sum \text { Wasser }^{\mathrm{a}} \text {, } \\
\text { Natur [kg] }\end{array}$ \\
\hline 2017 & 8 & 80 & 5,9 & 118,3 & 314,6 & 258,5 & 83,0 \\
\hline 2018 & 9 & 220 & 22,1 & 139,2 & 625,7 & 512,7 & 219,0 \\
\hline 2019 & 13 & 353 & 16,4 & 223,8 & $893,4^{b}$ & $695,3^{b}$ & - \\
\hline 2020 & 1 & 9 & 2 & 7 & 25,3 & 17,1 & 21,4 \\
\hline
\end{tabular}

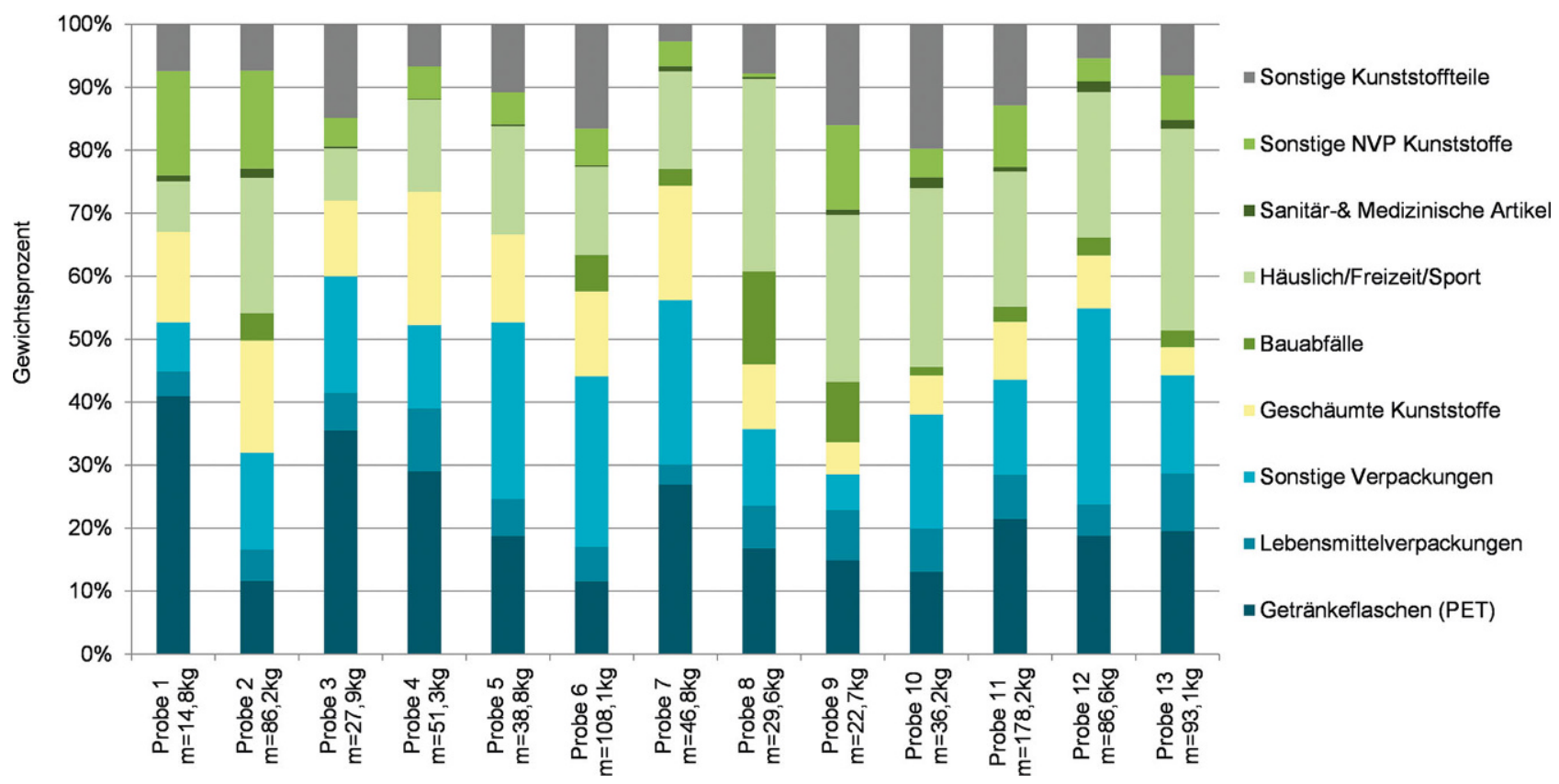

Abb. 2 Zusammensetzung der Kunststoffabfälle von Freiwilligen-Sammelkampagnen im NP Donau-Auen

packung (kein Brom) und Dämmstoffen getroffen werden.

\section{Ergebnisse}

\subsection{Sortierprotokoll}

Ein Pre-Test (erste Sortierungen) von Kunststoffabfällen aus dem Nationalpark Donau-Auen lieferte zunächst einen groben Überblick über die stoffliche Zusammensetzung der vorhandenen Kunststoffartikel. Darauf aufbauend wurde versucht, durch Zusammenfassung, Vereinfachung und Standardisierung ähnlicher (Produkt-)Gruppen bzw. Kategorien der Vorsortierung und der überprüften Protokolle eine vorläufige Kategorisierung von Kunststoffabfällen in Fließgewässern vorzunehmen. Aufgrund der projektspezifischen Frage nach der Herkunft der Kunststoffverschmutzungen wurde eine funktionale Klassifizierung gewählt, um die jeweiligen Fraktionen dem emittierenden Sektor bzw. der Quelle bestmöglich zu- ordnen zu können. Kunststoffobjekte oder -kategorien, die aufgrund ihrer chemischen Zusammensetzung, zugesetzten Additive oder physikalischen Struktur auf ein erhöhtes Umweltgefährdungspotenzial hinweisen, wurden zusätzlich auf Materialebene untergliedert.

Zurzeit sind 24 verschiedene Kategorien (siehe Tab. 2) definiert, die im Rahmen kontinuierlicher Sortieranalysen auf Nachvollziehbarkeit und Plausibilität geprüft und gegebenenfalls angepasst werden. Die gewählten Kategorien können auch von Laien ohne spezielle Vorkenntnisse bestimmt werden, um die Kunststoffzusammensetzung/Mengen ihrer gesammelten Abfälle $\mathrm{zu}$ vergleichen. Empfehlungen sowie Details zur korrekten Handhabung des Sortierprotokolls (https:// plasticfreeconnected.com/dow) werden in einem Handbuch festgehalten.

\subsection{Zusammensetzung der Kunststoffabfälle}

Über ein Jahr (Herbst 2017 bis Herbst 2018) wurden rund $940 \mathrm{~kg}$ reine Kunststoffabfälle aus den Hauptsammelstellen des Nationalparks Donau-Auen entfernt und anschließend sortiert. Im Durchschnitt sammelte jede Person 0,5 bis $2 \mathrm{~kg}$ Plastik pro Stunde. Bisher konnten neben anderen Abfällen rund 1,86 Tonnen reiner Plastikmüll aus dem Gebiet des Nationalparks im Zuge der Freiwilligensammlung entfrachtet werden. Tab. 2 gibt einen Überblick zu den bisherigen Sammelaktionen.

Die anschließenden Sortieranalysen lieferten erste Informationen über die Zusammensetzung dieser Kunststoffabfälle. Das untenstehende Diagramm (Abb. 2) zeigt die Ergebnisse von 13 Proben, die im Rahmen von Sammelaktionen durch Freiwillige entnommen wurden. Zur besseren Veranschaulichung wurden die einzelnen Kategorien in Untergruppen zusammengefasst. Die 
Untergruppen basieren auf der üblichen Zuordnung von erzeugten Kunststoffabfällen der Kunststoffindustrie (Plastics Europe 2018) sowie auf der zusätzlichen Identifizierung von Kategorien, die hinsichtlich ihres massen- und volumenbezogenen Auftretens explizit hervorgehoben wurden (bspw. Getränkeflaschen oder geschäumte Kunststoffe).

Die Ergebnisse der Sortieranalysen (Abb. 2) zeigen, dass PET-Getränkeflaschen und geschäumte Kunststoffe die beiden volumenmäßig größten Fraktionen sind, bei mehr als der Hälfte der Proben aber auch über 30 Gewichtsprozent ausmachen. Bei 7 der 13 Proben stellen PET-Flaschen die größte Kategorie dar, bei den übrigen gehören sie mindestens $\mathrm{zu}$ den Top 3. Auch die geschäumten Kunststoffe gehören mit 10 Proben zu den Top 4. Derzeit laufende Versuche zur genaueren Unterscheidung der Schaumstoffe (XRFMessungen) lassen darauf schließen, dass es sich vermehrt um geschäumte Dämmstoffe handelt.

Neben hausmüllähnlichen Abfällen sowie Sport- und Freizeitabfällen (rund $20 \%$ ) hat die Untergruppe der „Sonstigen Kunststoffteile“, meist sind dies nicht zuordenbare oder nicht-identifizierbare Bruchstücke, mit durchschnittlich $10 \%$ einen bedeutenden Anteil. Die "Nichtverpackungskunststoffe“ unterliegen teils großen Schwankungen, abhängig davon, ob bspw. schwerere Schifffahrtsabfälle wie Fender, Bojen o. Ä. gefunden wurden. Insgesamt konnte annähernd die Hälfte der gesammelten Kunststoffe der Hauptgruppe Verpackung zugeordnet werden (Anteil der Schaumstoffe ist dabei noch nicht berücksichtigt)

Bezüglich Standort und Sammelgebiet konnten vorerst keine Korrelationen festgestellt werden. Auch die Abfallzusammensetzung unterliegt keinen saisonalen Schwankungen (Sammlung Herbst/Frühwinter vs. Frühjahr/ Frühsommer), lediglich die Kategorie „Spiel-, Freizeit- und Sportartikel“ ist im Sommer durchschnittlich um sechs Prozentpunkte höher. Dies könnte auf den vermehrten Einsatz solcher Artikel in den Sommermonaten zurückzuführen sein.

\section{Diskussion und Schlussfolgerungen}

Ein Ziel des Projekts war es, die Herkunft der Kunststoffe zu bestimmen.
Die Analysen haben gezeigt, dass die gesammelten Kunststoffe im Gebiet des Nationalparks Donau-Auen nicht direkt abgelagert werden, sondern vielmehr über den Fluss als Treibgut von flussaufliegenden Verschmutzungsstellen angeschwemmt werden. Die $\mathrm{Zu}$ ordnung der eigentlichen Herkunft der Kunststoffe, z. B. nach direktem Littering (achtloses Wegwerfen in der Natur) oder sekundär (indirekt) über Wind oder beispielsweise Krähen verfrachtete Teile, die ursprünglich ordnungsgemäß in Abfallbehältern entsorgt wurden, ist nicht möglich. Trotzdem kann ein Großteil der Kunststoffe dem privaten Bereich zugeordnet werden. Allein rund $50 \%$ der Kunststoffe sind unterschiedliche Verpackungen, und davon wieder mehr als die Hälfte Lebensmittelverpackungen, was ein direktes Littering bei Picknicks, Grillereien etc. oder unsachgemäße Entsorgung vermuten lässt. Analysen haben gezeigt, dass eine vermehrte Anzahl an Abfallbehältern nicht unbedingt zu einer Verbesserung der Situation führt, obwohl laut einer Studie von Van der Meer et al. (2018) hervorgeht, dass die drei am häufigsten genannten Anti-Littering-Maßnahmen, welche als „sehr geeignet“ von der Bevölkerung eingeschätzt wurden, die Maßnahmen „mehr Abfallbehälter“, „gut sichtbares Platzieren der Abfallbehälter“ und „eine häufige Leerung der Abfallbehälter" darstellen. Van der Meer et al. (2018) zeigen jedoch, dass trotz einer geringen Distanz von zwei bis zehn Metern zu einem Abfallbehälter vermehrt gelittert wird. Das in der Literatur häufig genannte Argument, dass es keine Entsorgungsmöglichkeiten gäbe, wird somit entwertet. Von vielen ExpertInnen wird Umwelterziehung als die Maßnahme mit dem höchsten Potenzial angesehen, dem Littering Problem entgegenzuwirken (FUWA 2013; Neubert 2007).

Breitbarths (2017) Sortierkatalog von Makro-Kunststoffabfällen in Fließgewässern Deutschlands, der einen ähnlichen Aufbau, jedoch eine nicht zu detaillierte Klassifizierung aufweist, eignet sich durchaus für eine Gegenüberstellung der Ergebnisse aus dem Nationalpark Donau-Auen. Der Vergleich zeigt den signifikanten Einfluss eines Pfandsystems auf die Vermüllung von Kunststoff-Getränkeflaschen. Beispielsweise beträgt der Anteil pfandfreier Kunststoffflaschen in der Saale $0,8 \mathrm{Ge}$ wichtsprozent und 0,2 Gewichtsprozent pro Artikel, wobei $90 \%$ aller gefunde- nen Flaschen pfandfrei waren. Messungen in den Flüssen Fulda, Rhein und Haune zeigten ähnliche Ergebnisse (Breitbarth 2017). Umgekehrt machen im österreichischen Projektgebiet des Nationalparks Donau-Auen PETGetränkeflaschen in fast allen Sammlungen den größten Anteil aus (Masse 12 bis $40 \%$, aber auch Volumen). Laut Breitbarth kann das bestehende Pfandsystem in Deutschland bereits als eine wirksame Maßnahme zur Vermeidung von Littering bei Getränkeverpackungen angesehen werden.

Auf der Grundlage der Zusammensetzung der gesammelten und sortierten Kunststoffabfälle sowie der Erfahrungen der Nationalpark-Ranger können folgende vorläufige Schlussfolgerungen gezogen werden: (i) die Mehrheit der gesammelten Makro-Kunststoffabfälle ist auf direktes oder indirektes Littering zurückzuführen und (ii) der Großteil der Abfälle - als Treibgut aus Wien kommend - wird aus der Donau in die Umgebung ausgetragen. Regelmäßige Inspektionen und Kontrollen im Nationalpark haben gezeigt, dass die BesucherInnen wenig bis keinen Abfall im Erholungsgebiet hinterlassen. Gezielte Sensibilisierungsmaßnahmen in den letzten Jahren, wie z. B. die Reduktion der bestehenden Abfallbehälter entlang beliebter Wege und Freizeitoder Erholungsflächen, haben zu einer deutlichen Reduktion des Abfallaufkommens geführt. Die BesucherInnen wurden erfolgreich für die Mitnahme ihrer Abfälle sensibilisiert.

Wie bereits erwähnt, haben Maßnahmen wie die Einführung eines Pfands auf Einweg-Getränkeflaschen positive Auswirkungen auf das Littering- und Abfallaufkommen. Dies wird in Österreich derzeit im Zusammenhang mit der EU-Kunststoff-Einwegrichtlinie (Single-Use-Plastic- oder SUP-Richtlinie) diskutiert: Bis 2025 müssen demnach $77 \%$ der Getränkeflaschen aus Kunststoff gesammelt werden, bis 2029 sogar $90 \%$ (Europäische Kommission 2019). Derzeit werden in Österreich etwa $70 \%$ der ausgegebenen Flaschen gesammelt (Hauer et al. 2020). Eine Studie im Auftrag des BMK, die Umsetzungsmöglichkeiten der SUPRichtlinie anführt, zeigt auf, dass Österreich die $90-\%$-Sammelquote ohne Einführung eines Einweg-Pfands nur schwer erreichen wird (Hauer et al. 2020).

Auch die generelle Erhöhung der EU-Recyclingquoten für Kunststoffe im 
Rahmen des Europäischen Kreislaufwirtschaftspakets lässt für die Zukunft hoffen, dass sich die Abfallsituation verbessert und weniger Kunststoffabfälle in die Flüsse gelangen.

Danksagung Das Projekt PlasticFreeDanube wurde finanziert durch die Mittel des European Regional Development Fund Interreg V-A Slovakia - Austria 2014-2020 und kofinanziert durch das Bundesministerium für Landwirtschaft, Regionen und Tourismus (BMLRT), die Wiener Umweltschutzabteilung (MA 22), die Magistratsabteilung Wiener Gewässer (MA45) sowie die Verpackungskoordinierungsstelle GmbH (VKS).
Funding Open access funding provided by University of Natural Resources and Life Sciences Vienna (BOKU).

\section{Interessenkonflikt}

J. Mayerhofer, S. Lenz und G. Obersteiner geben an, dass kein Interessenkonflikt besteht.

Open Access Dieser Artikel wird unter der Creative Commons Namensnennung 4.0 International Lizenz veröffentlicht, welche die Nutzung, Vervielfältigung, Bearbeitung, Verbreitung und Wiedergabe in jeglichem Medium und Format erlaubt, sofern Sie den/die ursprünglichen Autor(en) und die Quelle ordnungsgemäß nennen, einen Link zur Creative Commons Lizenz beifügen und angeben, ob Änderungen vorgenommen wurden.

Die in diesem Artikel enthaltenen Bilder und sonstiges Drittmaterial unterliegen ebenfalls der genannten Creative Commons Lizenz, sofern sich aus der Abbildungslegende nichts anderes ergibt. Sofern das betreffende Material nicht unter der genannten Creative Commons Lizenz steht und die betreffende Handlung nicht nach gesetzlichen Vorschriften erlaubt ist, ist für die oben aufgeführten Weiterverwendungen des Materials die Einwilligung des jeweiligen Rechteinhabers einzuholen.

Weitere Details zur Lizenz entnehmen Sie bitte der Lizenzinformation auf http://creativecommons.org/licenses/ by/4.0/deed.de.
Breitbarth, M. (2017): Abfälle in deutschen Fließgewässern - Eintrags- und Austragspfade, Zusammensetzung, Aufkommen und Vermeidungsmaßnahmen. Schriftenreihe des Fachgebietes Abfalltechnik, Band 22.

Cheshire, A., Adler, E., Barbière, J., Cohen,

Y., Evans, S., Jarayabhand, S., Westphalen, G. (2009): UNEP/IOC Guidelines on Survey and Monitoring of Marine Litter.

European Commission (2019): Circular Economy: Commission welcomes European Parliament adoption of new rules on single-use plastics to reduce marine litter. Verfügbar in: http:// europa.eu/rapid/press-release_STATEMENT19-1873_en.htm. Zugegriffen: 06.07.2020

FUWA (2013): Handbuch zur Durchführung von Awarenesskampagnen im Bereich Abfallvermeidung, Abfalltrennung und Littering. Wien: Future of Waste (FUWA).

Hauer, W., Beigl, P., Allesch, A. et al. (2020): Möglichkeiten zur Umsetzung der EU-Vorgaben betreffend Getränkegebinde, Pfandsystem und Mehrweg. Studie im Auftrag des des $\mathrm{Mi}$ nisteriums für Klimaschutz, Umwelt, Energie, Mobilität, Innovation und Technologie (BMK).

IMO, FAO, UNESCO, WMO, WHO, IAEA, UN, UNEP - Joint Group of Experts on the Scientific Aspects of Marine Pollution (GESAMP). (1990): The state of the marine environment. Rep. Stud. GESAMP No. 39, pp. 111. London.
Jambeck, J. R., Geyer, R., Wilcox, C., Siegler, T. R., Perryman, M., Andrady, A., Law, K. L. (2015): Plastic waste inputs from land into the ocean. Science, 347. https://doi.org/10.1126/science. 1260352

Lebreton, L. C. M., Van Der Zwet, J., Damsteeg, J. W., Slat, B., Andrady, A., \& Reisser, J. (2017): River plastic emissions to the world's oceans. Nature Communications, 8. https://doi.org/10. 1038/ncomms 15611

Lechner, A., Keckeis, H., Lumesberger-Loisl, F., Zens, B., Krusch, R., Tritthart, M., Schludermann, E. (2014): The Danube so colourful: A potpourri of plastic litter outnumbers fish larvae in Europe's second largest river. Environmental Pollution, 188, 177-181. https://doi.org/10. 1016/j.envpol.2014.02.006

Neubert, K. (2007): Ethik in der Abfallwirtschaft - ethische und psychologische Ansätze zur Lösung des Littering-Problems. Herrsching: Bayrisches Landesamt für Umwelt - Abteilung Abfallwirtschaft.

NPDA (2011): The Donau-Auen National Park. Verfügbar in: https://www.donauauen.at/dernationalpark/. Zugegriffen: 06.07.2020 OSPAR (2010): Guideline for Monitoring Marine Litter on the Beaches in the OSPAR Maritime Area (Vol. 1). London: OSPAR Commission. PlasticsEurope (2018): Plastics-The facts 2018: An analysis of European plastics production, demand and waste data. Brussels-Belgium.
Schmidt, C., Krauth, T., \& Wagner, S. (2017): Export of Plastic Debris by Rivers into the Sea. Environmental Science \& Technology, 51(21), 12246-12253. https://doi.org/10.1021/acs.est. 7 b02368

Van der Meer, E.; Beyer, R. und Gerlach, R. (2018): Wahrnehmung von Sauberkeit und Ursachen von Littering. Berlin: Verband kommunaler Unternehmen e. V..

Vannela, R. (2012): Are We "Digging Our Own Grave" Under the Oceans? Biosphere-Level Effects and Global Policy Challenge from Plastic(s) in Oceans. Environmental Science \& Technology, 46, 7932-7933. https://doi.org/10.1021/ es302584e

van der Wal, M., van der Meulen, M., Tweehuijsen, G., Peterlin, M., Palatinus, A., Kovac Virsek, M., Krzan, A. (2015): Final Report on Identification and Assessment of Riverine Input of (Marine) Litter. Bristol—United

Hinweis des Verlags Der Verlag bleibt in Hinblick auf geografische Zuordnungen und Gebietsbezeichnungen in veröffentlichten Karten und Institutsadressen neutral. 